\title{
Behavioral and electrophysiological effects of transcranial direct current stimulation of the parietal cortex in a visuo-spatial working memory task
}

\author{
K. Heimrath ${ }^{1}$, P. Sandmann ${ }^{2}$, A. Becke ${ }^{3}$, N. G. Müller ${ }^{3}$ and T. Zaehle ${ }^{1 *}$ \\ 1 Section of Neuropsychology, Department of Neurology, Otto-von-Guericke University Magdeburg, Magdeburg, Germany \\ 2 Neuropsychology Lab, Department of Psychology, Carl von Ossietzky University of Oldenburg, Oldenburg, Germany \\ ${ }^{3}$ German Centre for Neurodegenerative Diseases, Magdeburg, Germany
}

\section{Edited by:}

Alberto Priori, Università di Milano, Italy

Reviewed by:

John Hart, University of Texas at Dallas, USA

David Luck, Université de Montréal, Canada

${ }^{*}$ Correspondence:

T. Zaehle, Section of

Neuropsychology, Department of Neurology, Otto-von-Guericke

University Magdeburg, Leipziger

Strasse 44, 39120 Magdeburg,

Germany.

e-mail: tino.zaehle@ovgu.de
Impairments of working memory (WM) performance are frequent concomitant symptoms in several psychiatric and neurologic diseases. Despite the great advance in treating the reduced WM abilities in patients suffering from, e.g., Parkinson's and Alzheimer's disease by means of transcranial direct current stimulation (tDCS), the exact neurophysiological underpinning subserving these therapeutic tDCS-effects are still unknown. In the present study we investigated the impact of tDCS on performance in a visuo-spatialWM task and its underlying neural activity. In three experimental sessions, participants performed a delayed matching-to-sample WM task after sham, anodal, and cathodal tDCS over the right parietal cortex. The results showed that tDCS modulated WM performance and its underlying electrophysiological brain activity in a polarity-specific way. Parietal tDCS altered eventrelated potentials and oscillatory power in the alpha band at posterior electrode sites. The present study demonstrates that posterior tDCS can alter visuo-spatialWM performance by modulating the underlying neural activity. This result can be considered an important step toward a better understanding of the mechanisms involved in tDCS-induced modulations of cognitive processing. This is of particular importance for the application of electrical brain stimulation as a therapeutic treatment of neuropsychiatric deficits in clinical populations.

Keywords: tDCS, TES, working memory, parietal cortex, EEG, working memory capacity, alpha

\section{INTRODUCTION}

Working memory (WM) refers to a mental workspace that allows one to temporally store and manipulate a limited amount of information in mind. WM functioning is essential for a wide range of complex cognitive tasks, such as reasoning, problem solving, language comprehension, and learning (Baddeley, 1992). WM performance typically activates a fronto-parietal network, including the dorsolateral prefrontal cortex (DLPFC; Smith and Jonides, 1997; Courtney et al., 1998; Nystrom et al., 2000; Hautzel et al., 2002) and the posterior parietal lobe (Owen et al., 2005). While the DLPFC is involved in the processing of stimulus information during retention times (Funahashi et al., 1993), the parietal cortices are responsible for the storage of perceptual attributes (Callicott et al., 1999), and the maintenance of information specifically regarding spatial locations (Olson and Berryhill, 2009) hereby constituting the capacity limit for the amount of items an individual is able to store (Todd and Marois, 2004). Consequently, the involvement of the posterior parietal lobe is consistently found during a wide range of WM tasks (Wager and Smith, 2003). Accordingly, damage of the posterior parietal lobe leads to WM impairments (Olson and Berryhill, 2009).

Impairments of WM performance are frequent concomitant symptoms in several psychiatric and neurologic diseases. Patients suffering from Alzheimer's disease (AD) exhibit specific deficits in visual and spatial WM performance. They demonstrate serious impairments of spatial memory span and the retention of visual information (Huntley and Howard, 2010). Specifically, the spatial WM component seems to be more strongly affected in $\mathrm{AD}$ compared to individuals with mild cognitive impairment (AlescioLautier et al., 2007). Likewise, patients with Parkinson's disease (PD) demonstrate a remarkable reduction in visuo-spatial WM abilities (Lees and Smith, 1983). In particular, these patients exhibit diminished storage capacities accompanied with deficits in retaining spatial, visual, and verbal information (Owen et al., 1997; Lee et al., 2010). Moreover, patients with amyotrophic lateral sclerosis (ALS) show reduced WM abilities associated with reduced spatial WM capacity (Hammer et al., 2011). In addition to neurological patient populations, psychiatric patients widely display specific WM impairments such as comprehensive visual WM abnormalities in patients with schizophrenia (Barch et al., 2003) and depression (Rose and Ebmeier, 2006).

Even though the administration of dopamine agonists has been shown to improve WM in normal subjects and patients with traumatic brain injury (TBI; McAllister et al., 2011; Wallace et al., 2011), the success of pharmacological treatments of WM-deficits is still restricted in terms of limited effectiveness and side-effects (Birks, 2006; Marder, 2006; McGurk et al., 2007). In recent years, transcranial direct current stimulation (tDCS) has been employed as a new approach to alter memory performances in healthy participants as well as to improve abnormal memory abilities 
in neuropsychiatric patients (Brunoni et al., 2011; Nitsche and Paulus, 2011).

Transcranial direct current stimulation is a non-invasive technique for delivery of low currents to the cerebral cortex that results in the modulation of cortical excitability (Bindman et al., 1962). With tDCS, weak constant electric currents are applied on the cortical surface in a non-invasive and painless manner (Priori, 2003; Fox, 2011). The current flows between an active and a reference electrode. While a part of this current is shunted through the scalp, the rest is delivered to the brain tissue (Miranda et al., 2006), thereby inducing diminutions or enhancements of cortical excitability (Nitsche et al., 2008). The direction of the tDCSinduced effect depends on the current polarity. Anodal tDCS typically has an excitatory effect while cathodal tDCS decreases the cortical excitability in the region under the electrode (Nitsche and Paulus, 2000; Nitsche et al., 2003). Specifically, anodal tDCS causes a depolarization of the resting membrane potential and increases the firing rate, whereas cathodal tDCS decreases the firing rate via hyperpolarization of the resting membrane potential (Bindman et al., 1962; Purpura et al., 1965). Importantly, tDCS effects are not restricted to this primary polarization mechanism during stimulation, because after-effects persist over minutes to hours. These after-effects of tDCS are associated with a number of different mechanisms, including local changes in ionic concentrations (hydrogen, calcium) and levels of cyclic adenosine monophosphate (cAMP; Hattori et al., 1990), alterations in protein synthesis, and modulation of $N$-methyl-D-aspartate (NMDA) receptor efficacy (Gartside, 1968; Hattori et al., 1990; Liebetanz et al., 2002).

To date, the neuromodulatory changes induced by tDCS have been associated with modifications of the motor (Priori et al., 1998; Nitsche and Paulus, 2000) as well as a variety of sensory systems, including the visual (Antal et al., 2003, 2004; Accornero et al., 2007), the somatosensory (Dieckhofer et al., 2006; Antal et al., 2008), and the auditory system (Vines et al., 2006; Loui et al., 2010; Zaehle et al., 2011a). In the cognitive domain, polarityspecific effects of tDCS have been reported for WM functions in healthy participants (Fregni et al., 2005; Ohn et al., 2008; Zaehle et al., 2011b). Anodal tDCS over the DLPFC improves visual WM (Fregni et al., 2005; Zaehle et al., 2011b), whereas cathodal stimulation of the DLPFC interferes with short-term auditory memory performance (Elmer et al., 2009) and cathodal tDCS over the right inferior parietal cortex impairs object recognition WM (Berryhill et al., 2010). Regardless of polarity, tDCS over the cerebellum disrupts practice-dependent improvement during a verbal WM performance (Ferrucci et al., 2008b), whereas bifrontal tDCS impairs response selection and preparation in a verbal WM task (Marshall et al., 2005).

Most importantly, in addition to the progress of tDCS-related treatments of several cognitive (Fregni et al., 2006; Monti et al., 2008), affective (Boggio et al., 2008; Nitsche et al., 2009), and motor (Boggio et al., 2007; Bolognini et al., 2009) symptoms in neuropsychiatric disorders, first successful attempts in the direct modulation of specific memory deficits in neuropsychiatric patient populations have been demonstrated (Brunoni et al., 2011). It has been shown that idiopathic Parkinson patients could increase accuracy in a three-back letter WM task by approximately $20 \%$ during 20 min of $2 \mathrm{~mA}$ anodal tDCS over the left DLPFC (Boggio et al., 2006). Analogously, in stroke patients Jo et al. (2009) demonstrated enhanced recognition accuracy by approximately $10 \%$ in a verbal WM task after $30 \mathrm{~min}$ of $2 \mathrm{~mA}$ anodal tDCS over the left DLPFC. Moreover, in patients with AD 30 min anodal tDCS at $2 \mathrm{~mA}$ over left temporal and left DLPFC could increase recognition memory by 18.03 and $13.8 \%$, respectively (Boggio et al., 2009). Similarly, in this patients word recognition memory could be improved by approximately $15 \%$ after $15 \mathrm{~min}$ anodal tDCS at $1.5 \mathrm{~mA}$ over tempo-parietal regions (Ferrucci et al., 2008a). Finally, bilateral anodal tDCS over the temporal cortex of AD patients at five consecutive days improves the visual recognition memory by $8.99 \%$ for at least 4 weeks (Boggio et al., 2011). However, the nature of the neurophysiological mechanisms underlying this tDCS-related cognitive enhancement is not well understood. In a recent study, we investigated the impact of tDCS over the left DLPFC on performance in a WM task and its underlying neural activity in healthy participants (Zaehle et al., 2011b). The anodal tDCS improved, whereas cathodal tDCS interfered with WM performance. These tDCS-induced effects were reflected in the neural oscillatory activity, showing polarity-specific alterations as a function of tDCS. Anodal tDCS enhanced, whereas cathodal tDCS suppressed the event-related oscillatory power in the theta and alpha range.

In the present study we examined the impact of tDCS on visuo-spatial WM performance and the underlying neural activity. In particular, we explored the effect of tDCS applied over the right parietal lobe on electrophysiological brain activity during a delayed matching-to-sample WM task. Given the critical involvement of the posterior parietal lobe in WM functions (Todd and Marois, 2004; Corbetta et al., 2008; McNab and Klingberg, 2008), we hypothesized tDCS-dependent alteration of WM performance. Furthermore, we predicted tDCS-related modifications of the underlying neural activity. To our knowledge, this is the first study to investigate the modulatory effects of parietal tDCS on electrophysiological brain activity in the context of a visuo-spatial WM task.

\section{MATERIALS AND METHODS PARTICIPANTS}

Twelve healthy adults participated in this study (seven female). The age range was 21-31 years (mean 25.8 years). All subjects reported being consistent right-handers, having no metallic implant, no history of neuropsychiatric disorder, and normal or corrected-to-normal vision acuity and color vision.

\section{TRANSCRANIAL DIRECT CURRENT STIMULATION}

Participants were seated comfortably in a recliner in front of a personal computer screen in an electromagnetically shielded room. The current was applied by a battery-driven DC-stimulator (Eldith, NeuroConn GmbH, Germany) using a pair of rubber electrodes in $5 \times 7 \mathrm{~cm}$ synthetic sponges soaked in $0.9 \% \mathrm{NaCl}$ solution. For stimulation of the right parietal cortex the active electrode (to which the term anodal/cathodal stimulation refers) was placed over P8/P10 and the reference electrode over P7/P9. These electrode positions were consistent to the European 10 20 system for electroencephalography (EEG) electrode placement 
(Jasper, 1958). Each participant performed three separate tDCS sessions: one anodal, one cathodal, and one sham tDCS session separated by at least $24 \mathrm{~h}$ to avoid carry-over effects. The session order was counterbalanced across participants. Within each session, a constant current of $1 \mathrm{~mA}$ was applied for $30 \mathrm{~min}$, with a linear fade in/fade out of $10 \mathrm{~s}$. For sham stimulation the electrodes were placed on same positions, but after a fade in period of $10 \mathrm{~s}$ the stimulator was turned off without awareness of the participants. This procedure ensured that in the sham and stimulation conditions, participants experienced the initial itching that recedes over the first seconds of tDCS. Accordingly, none of the participants were able to determine whether or not they received real or sham stimulation.

\section{WORKING MEMORY ASSESSMENT}

The procedure of the experimental sessions was carried out sequentially: the participants performed a delayed matching-tosample visuo-spatial WM task (Vogel and Machizawa, 2004) with concurrent EEG recording starting $7.4 \mathrm{~min} \pm 2.4(\mathrm{SD})$ after each tDCS condition (sham, anodal, cathodal). Stimulus presentation was controlled by the Presentation software (Neurobehavioral Systems, USA). During each trial, subjects were presented a fixation cross $(2800 \pm 3500 \mathrm{~ms})$ followed by an arrow $(200 \mathrm{~ms})$ indicating the hemifield (left/right) to be attended. A memory array was then presented within two rectangular regions that were centered to the left and right on a gray background. These two rectangular regions of the memory arrays consisted of four colored circles $\left(0.69^{\circ}\right)$ with randomized position (within a rectangle) and were randomly colored (blue, brown, green, red, cyan, yellow, orange, pink, black, white). The memory array appeared for $150 \mathrm{~ms}$ and was followed by a retention period of $2000 \mathrm{~ms}$ during which subjects had to retain the memory array. This was followed by the presentation of a test array with one circle in the center of the screen, which was either identical or different in color compared to the circles shown in the memory array (cf. Figure 1). Subjects had $2000 \mathrm{~ms}$ before the onset of the next trial to make a push-button response to indicate whether or not the probe stimulus in the test array was identical to one stimulus in the memory array. The test sequence consisted of 256 trials separated into four runs. The order of the trials was

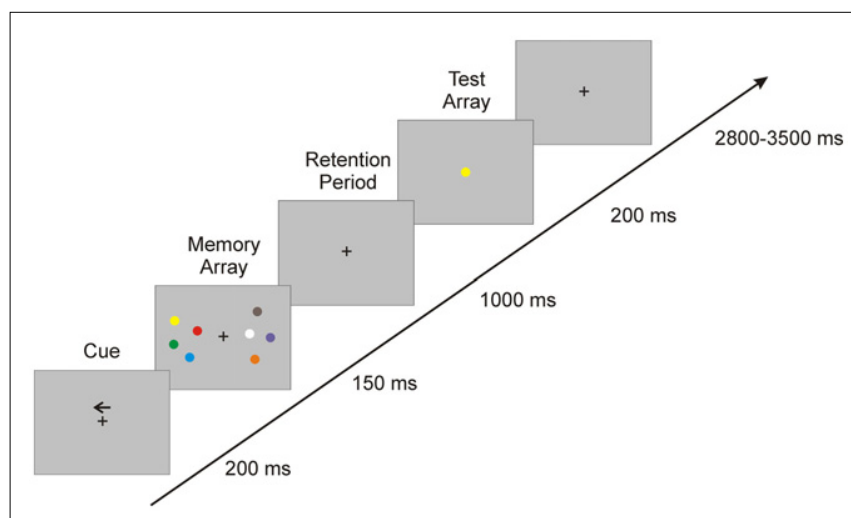

FIGURE 1 | Experimental design: the figure illustrates the sequence of events in each trial. identical across individual sessions but pseudo randomized across subject.

To assess the individual WM performance, we calculated the WM capacity $K$ (Cowan's coefficient; Cowan, 2001) for each tDCS stimulation condition (sham, anodal, cathodal). Values for $K$ were estimated for each subject by $K=S(H-F)$. The formula assumes, that if $K$ items can be held in WM from an array of $S$ objects, the probed item would have been one of those held in memory on $K / S$ of the trials such that performance will be correct on $\mathrm{K} / \mathrm{S}$ of the change trials ( $=$ hit rate $H$ ). To correct for guessing, this procedure also takes into account the false alarm rate $F$.

In the end, $K$ values were analyzed using $3 \times 2$ repeatedmeasures ANOVAs with the within-subject factor tDCS (sham, anodal, and cathodal) and attended hemifield (left, right). Greenhouse-Geisser correction was applied in case of violation of the sphericity assumption.

\section{EEG RECORDING AND ANALYSIS}

During the WM task, EEG was recorded from 19 standard scalp locations according to the European 10-20 system (Fp1, Fp2, F3, F4, F7, F8, Fz, Cz, C3, C4, T3, T4, Pz, P3, P4, T5, T6, O1, O2) using $\mathrm{Ag} / \mathrm{AgCl}$ electrodes mounted in an elastic cap (Soft Cap EEGH-Z-*, Walter Graphtec GmbH). The vertical and horizontal electrooculogram was recorded with one electrode placed below and one placed approximately $1 \mathrm{~cm}$ to the external canthus of the right eye. EEG data were recorded by a PL-351 amplifier and the corresponding software (Walter Graphtek $\mathrm{GmbH}$ ) referenced to electrode $\mathrm{POz}$ and sampled at $500 \mathrm{~Hz}$. Impedances were kept below $10 \mathrm{k} \Omega$. EEG preprocessing and data analysis were carried out in Brain Vision Analyzer 2.0 (Brain Products, Munich, Germany), and FieldTrip http://fieldtrip.fcdonders.nl/. EEG data were off-line filtered from 1 to $40 \mathrm{~Hz}$ and re-referenced to a common average reference. Event-related potentials (ERPs) were segmented into $1300 \mathrm{~ms}$ epochs starting $300 \mathrm{~ms}$ before the onset of the memory array and covered the retention period, thus analyzing the encoding and retention phase of the WM task. Baseline correction was accomplished between -300 and $-200 \mathrm{~ms}$. Segments containing ocular artifacts, movement artifacts, or amplifier saturation were excluded from the averaged ERP waveforms. ERPs for each stimulus (attend left, attend right, separately for sham, anodal, and cathodal) were averaged for each subject and grand-averaged across subjects.

Subsequently, for posterior channels (P3, P4, Pz, O1, O2) peak analysis of the ERP was performed on single-subject averages measured for the ERP components N2 (most negative deflection between 100 and $200 \mathrm{~ms}$ ), P2 (positive deflection between 180 and $280 \mathrm{~ms}$ ), and N3 (negative deflection between 240 and $340 \mathrm{~ms}$ ). Furthermore, the sustained posterior contralateral negativity (SPCN; Klaver et al., 1999) was investigated by calculating the mean amplitude in a latency range between 600 and $700 \mathrm{~ms}$. These latencies were defined on the basis of the grand average computed across all participants and conditions. In the end, amplitude measures were analyzed using separate repeated-measures ANOVAs. These $3 \times 2$ ANOVAs included the within-subject factor tDCS (sham, anodal, and cathodal) and attended hemifield (left and right). Greenhouse-Geisser correction was applied in case of violation of the sphericity assumption. 
Furthermore, event-related spectral perturbations (ERSP) were analyzed for each subject and condition. ERSPs were calculated for parietal and occipital channels (P3, P4, Pz, O1, O2) using a wavelet-based analysis implemented in Brain Vision Analyzer 2.0 software. We used a continuous wavelet transform (WT) with complex Morlet wavelets (Morlet parameter c 3.8; 40 frequency steps from 1 to $40 \mathrm{~Hz}$ ) to examine the frequency composition of single-trial epochs. The magnitudes of the WTs of single-trial epochs were then averaged to compute the total power of activity, which contains signal components that are phase-locked and non-phase-locked to the stimulus event. For each scale of the WT a baseline correction was applied by subtracting the mean amplitude within the -300 to $-50 \mathrm{~ms}$ time window from each data point after stimulus onset. tDCS effects on oscillatory brain activity were analyzed by computing ERSP differences between the separate tDCS conditions. For statistical comparisons, we used a non-parametric cluster-based randomization approach built into FieldTrip. All data points ( 40 frequency steps from 1 to $40 \mathrm{~Hz}$ ) were included in this global analysis of time frequency bands. In particular, this procedure defined clusters on the basis of the actual distribution of the data and tested the statistical significance of these clusters using a Monte-Carlo randomization method with correction for multiple comparisons (Maris et al., 2007). The clustering used 500 randomizations and was performed in time and frequency simultaneously. The $t$-statistic of paired $t$-tests was calculated on a cluster-level by taking the sum of the $t$-values within the respective cluster (Jacobson et al., 2012).

\section{RESULTS}

\section{BEHAVIORAL DATA}

The $K$ value (Cowan, 2001), an individual estimate of WM capacity, was calculated for each subject separately for each tDCS condition (cf. Figure 2). The $3 \times 2$ repeated-measures ANOVA with the factors $t D C S$ (sham, anodal, cathodal) and hemifield (left, right) revealed a significant $t D C S \times$ hemifield interaction $[F(1.8,20.4)=4.16, P<0.05]$. Neither the main effect for factor $t D C S$ [sham, anodal, cathodal; $F(1.9,21.6)=2.12, P=0.15$ ] nor the main effect for factor hemifield [left, right; $F(1.11)=0.54$; $P=0.48$ ] reached statistical significance. Subsequent separate ANOVAs with the factor $t D C S$ (sham, anodal, cathodal) for the left and right hemifield revealed a significant main effect of $t D C S$ for stimuli attended in the left hemifield only $[F(1.8,20.6)=3.93$, $P<0.05]$. While anodal tDCS of the right parietal cortex decreased WM capacity for contralateral stimuli, cathodal tDCS increased it. For stimuli that had to be attended on the ipsilateral (right) hemifield, both active tDCS conditions interfered with the WM capacity in comparison to the sham condition.

\section{EVENT-RELATED POTENTIALS}

Figure 3 illustrates the ERP data for anodal, cathodal, and sham conditions for stimuli attended in the left and right hemifield averaged over 12 subjects for the analyzed electrodes (P3, P4, Pz, O1, $\mathrm{O} 2$ ). Visual stimulation consistently evoked a N2 component at $150 \mathrm{~ms}$ which was followed by the P2 component at $230 \mathrm{~ms}$. A $\mathrm{N} 3$ component was elicited consistently in all tDCS conditions with a mean latency of $300 \mathrm{~ms}$ which was followed by an SPCN component between 600 and $700 \mathrm{~ms}$.

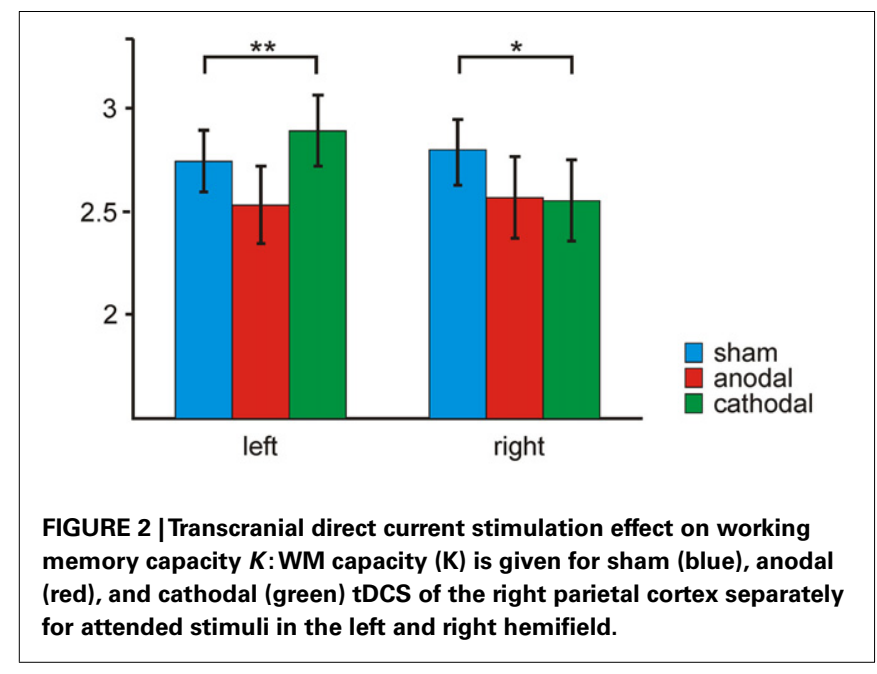

For the amplitude of the N2 ERP component the $3 \times 2$ repeated measurement ANOVAs with the factors tDCS (sham, anodal, cathodal) and hemifield (left, right) revealed a significant main effect of the factor $t D C S$ at electrodes $\mathrm{P} 3[F(1.9,22.2)=4.64$, $P<0.05]$, and $\mathrm{Pz}[F(1.9,21.4)=5.29, P<0.05]$, and a statistical trend at electrode $\mathrm{P} 4[F(1.5,16.9)=2.66, P=0.1]$. Anodal tDCS reduced the $\mathrm{N} 2$ amplitude as compared to sham and cathodal stimulation regardless of the attended hemifield in the bilateral posterior cortex (cf. Figure 4).

Analysis of the amplitude of the $\mathrm{P} 2$ component revealed a significant $t D C S \times$ hemifield interaction at electrodes P3 [F(1.6, $17.2)=8.82, P<0.01]$, and a statistical trend at $\mathrm{Pz}[F(1.6$, $17.1)=3.29, P=0.07]$. Subsequent separate ANOVAs with the factor $t D C S$ (sham, anodal, cathodal) revealed a significant main effect of the factor $t D C S$ for stimuli attended in the right hemifield at electrode $\mathrm{P} 3[F(1.8,20.4)=5.29, P<0.05]$. Both, anodal and cathodal tDCS decreased the P2 amplitude as compared to sham stimulation for stimuli attended in the right hemifield at left and central posterior electrode sites.

N3 amplitudes were modulated by tDCS at electrodes O1 $[F(1.7,19.2)=2.69, P=0.09]$ and $\mathrm{O} 2[F(1.9,20.9)=4.69$, $P<0.05]$. Both, anodal and cathodal tDCS decreased the N3 amplitude as compared to sham stimulation.

Statistical analysis of the SPCN revealed a significant $t D C S \times$ hemifield interaction at electrode $\mathrm{P} 3[F(1.8,19.7)=5.88$, $P<0.05]$, and a statistical trend at electrode O1 $[F(1.3$, $14.2)=2.68, P=0.09$ ]. Subsequent separate ANOVAs with the factor $t D C S$ revealed significant main effect of the factor $t D C S$ for stimuli attended in the left hemifield at electrode P3 [F(1.4, $15.8)=4.04, P<0.05]$, and for stimuli attended in the right hemifield at electrode $\mathrm{O} 1[F(1.5,17)=3.48, P=0.06]$. Both, anodal and cathodal tDCS decreased the SPCN amplitude over left posterior scalp regions for stimuli attended in the left hemifield, whereas active tDCS increased the amplitude for stimuli attended in the right hemifield over left occipital scalp regions.

\section{EVENT-RELATED SPECTRAL PERTURBATION}

Non-parametric cluster permutation statistics were computed on ERSPs to compare the different $t D C S$ conditions. This analysis was 


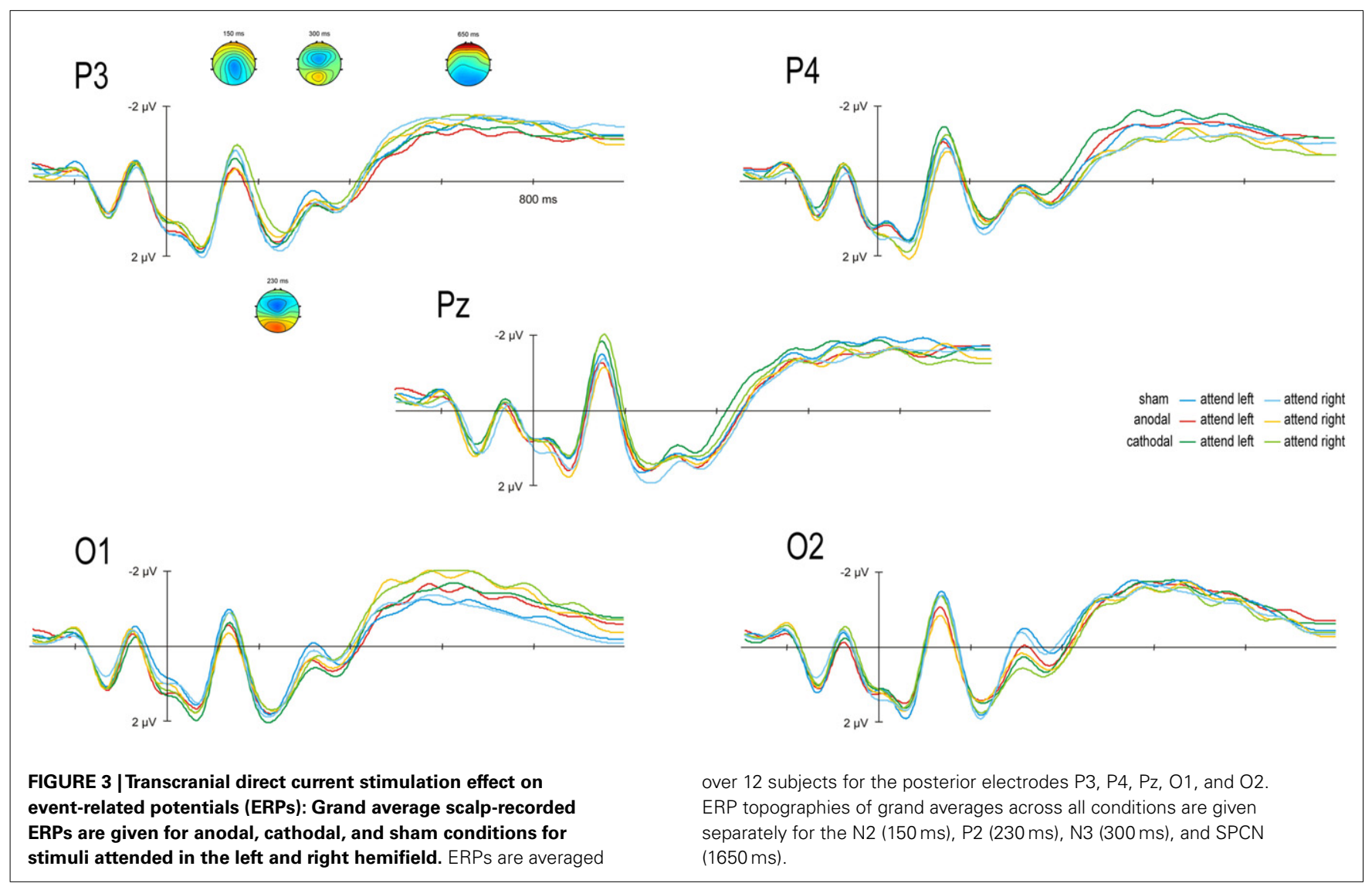

conducted separately for the attend left and attend right condition and revealed a significant decrease in the alpha band $(6-16 \mathrm{~Hz})$, at the latency range of $96-432 \mathrm{~ms}\left(t_{11} \geq 2.2, P<0.05\right)$ after cathodal as compared to anodal tDCS at electrode $\mathrm{Pz}$ for the attend right condition only. Figure 5 shows the ERSP time frequency plots for the sham condition and the active stimulation conditions (anodal, cathodal) plus the corresponding differences at electrode Pz.

\section{DISCUSSION}

This study investigated the impact of parietal tDCS on performance in a visuo-spatial WM task and its underlying neural activity. To achieve this goal, participants performed in three separate sessions under sham, anodal, and cathodal tDCS of the right parietal cortex a delayed matching-to-sample task, in which four visual stimuli presented in one visual field had to be memorized and compared with a single subsequently presented test stimulus.

Parietal tDCS during the visuo-spatial WM task had a significant modulatory effect on the WM capacity. Anodal tDCS over the right parietal lobe decreased WM capacity for stimuli attended in the left hemifield, whereas right parietal cathodal tDCS increased WM capacity for attended stimuli in the left hemifield. These modulations in WM capacity can be related to modulated activity in posterior brain areas during the execution of the WM task. Analysis of the ERP during memory encoding and retention revealed that specific ERP components are modulated by the active tDCS conditions. In particular, anodal tDCS generally reduced the $\mathrm{N} 2$ amplitude over the bilateral posterior cortex regardless of the attended hemifield, whereas both, anodal, and cathodal tDCS decreased the P2 amplitude for stimuli attended in the right hemifield at left and central posterior electrode sites. Furthermore, active $\mathrm{tDCS}$ decreased the N3 amplitude over bilateral occipital areas. The SPCN amplitude over left posterior scalp regions was reduced by active tDCS for stimuli attended in the left hemifield, whereas anodal and cathodal tDCS increased the amplitude for stimuli attended in the right hemifield over left occipital scalp regions. Furthermore, right parietal cathodal tDCS decreased event-related oscillatory power in the alpha band.

On the behavioral level, in the present study we found that cathodal tDCS improved the visuo-spatial WM capacity, whereas anodal tDCS slightly interfered with the WM performance when tDCS was applied over the right parietal cortex. Notwithstanding these results are in contrast to the commonly observed anodalimprovement/cathodal-impairment dichotomy, our tDCS-effects are consistent with recent studies demonstrating tDCS-related modulation of higher cognitive functions. Monti et al. (2008) found that cathodal tDCS over left fronto-temporal areas significantly improved the accuracy of picture naming, whereas anodal tDCS failed to induce any changes. Similarly, You et al. (2011) found that cathodal tDCS over right superior temporal areas induced significantly greater improvements in auditory verbal comprehension than anodal tDCS or sham tDCS over left superior temporal areas. Furthermore, Boggio et al. (2010) found that both, anodal and cathodal tDCS increased the propensity for risktaking. It has been suggested that the observed improvement after 


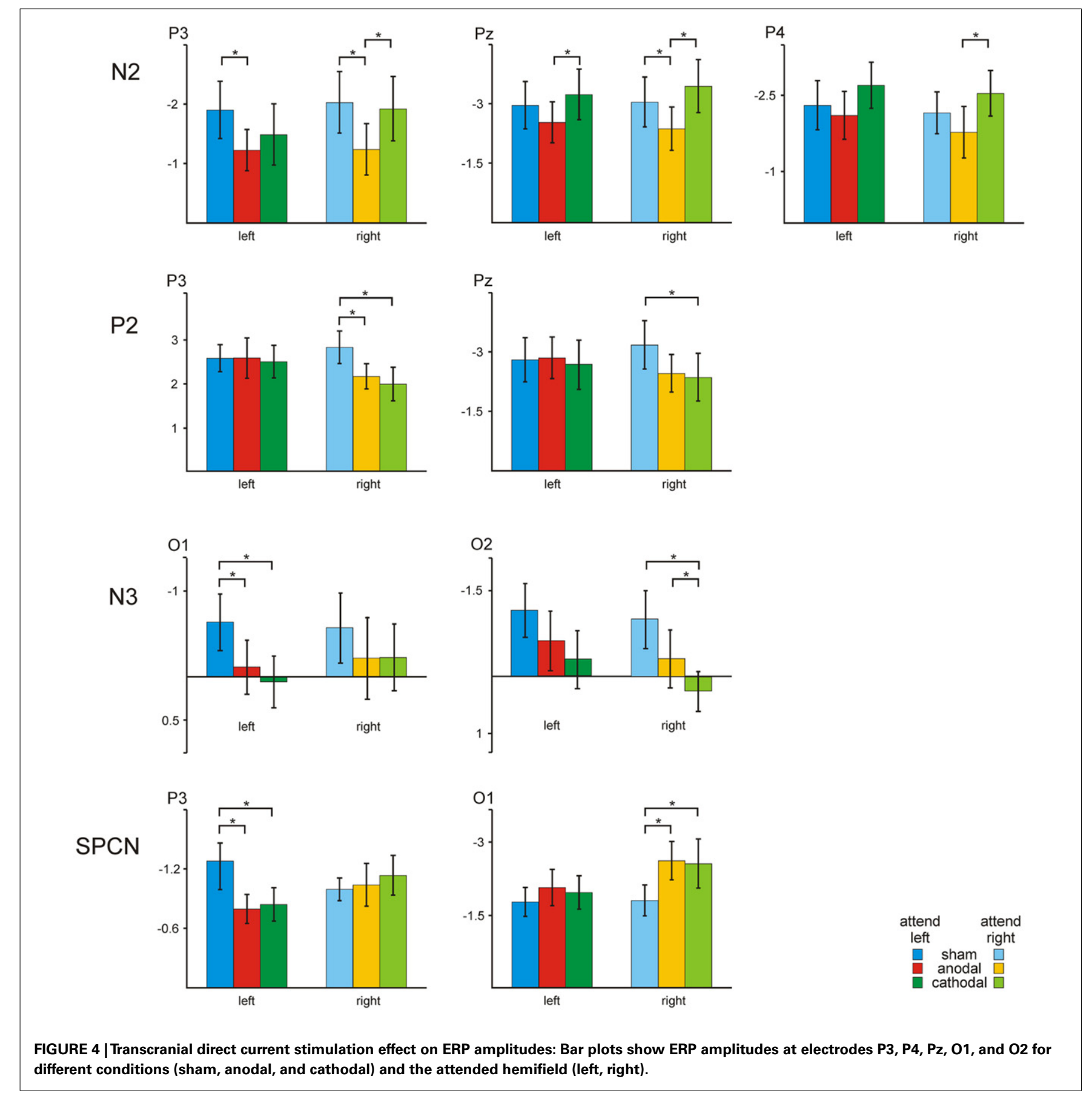

cathodal tDCS might be related to a tDCS-induced depression of cortical inhibitory interneurons, leading to a disinhibition, and, consequently, to an improved functioning of the target cortex (Monti et al., 2008). Generally, the commonly observed anodalimprovement/cathodal-impairment dichotomy is seen mainly in motor studies and rarely in cognitive studies (Jacobson et al., 2012). Furthermore, the distribution of the current flow through the head is much more complex and even common tDCS parameters cannot fully predict the current that reaches the cortex (Neuling et al., 2012). Therefore, in addition to the polarity of modulation, effects of tDCS on WM often depend on additional various factors, such as the task, current density, modulation duration, electrode montage, electrode size, and orientation of the electric field in relation to the anatomical and geometrical feature of the cortex. In the context of the current electrode mounting at parietal electrode sites P7/9, Neuling and colleagues could demonstrate that beside posterior (parietal and occipital) brain areas, also temporal, and frontal cortices reach current densities.

In this study we further assessed the electrophysiological brain activity during the WM task in order to investigate the 


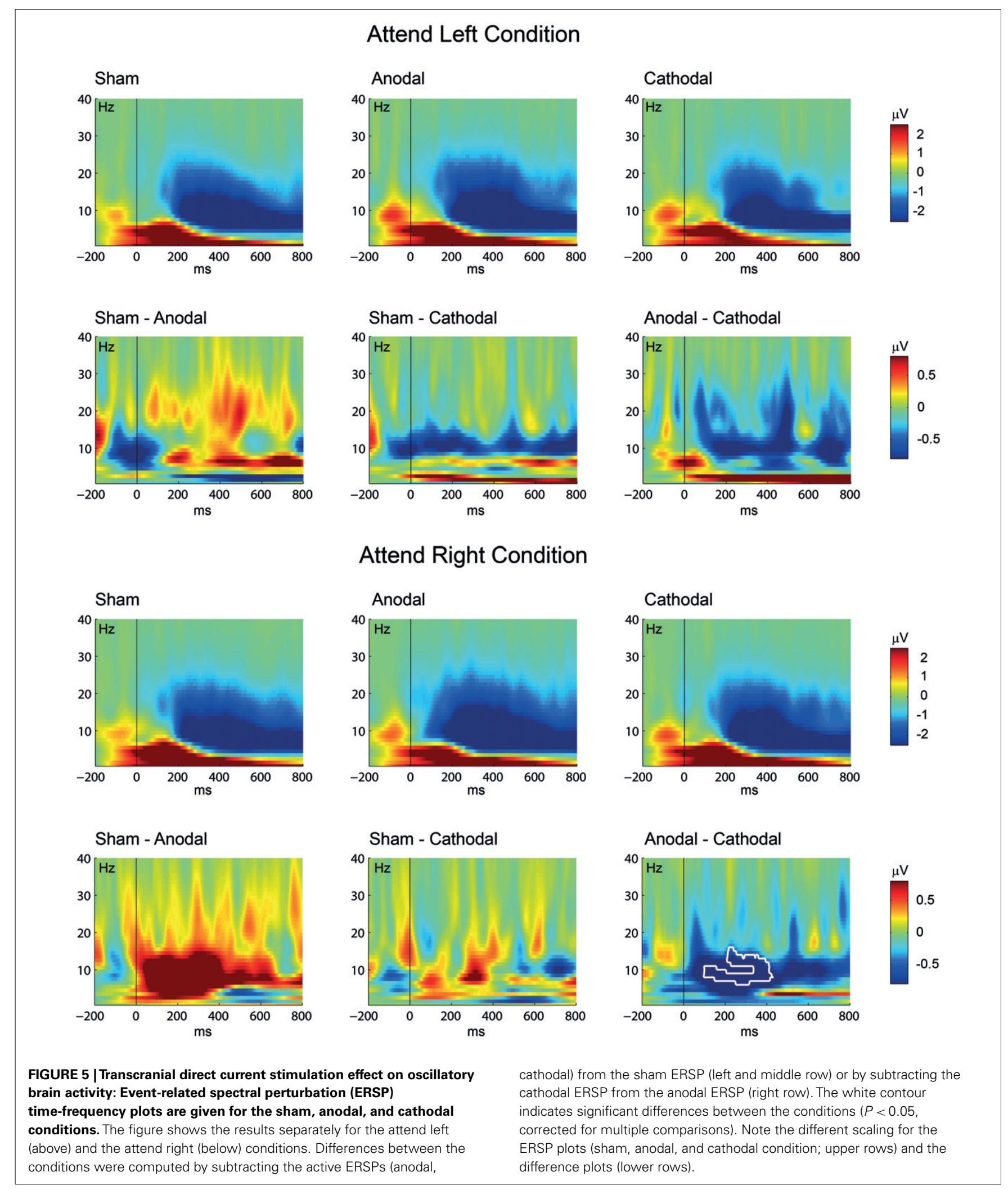

underlying neural mechanisms mediating the tDCS-induced behavioral effects. To date, reports of electrophysiological correlates of tDCS effects are sparse. Using visual ERPs, Antal et al.
(2004) demonstrated that the amplitude of the N70 ERP component is increased by anodal tDCS, while it is decreased by cathodal tDCS. The opposite effect has been reported for the visual P100, 
showing reduced amplitudes for anodal and increased amplitudes for cathodal stimulation (Accornero et al., 2007). Polarity-specific changes have also been observed for motor cortex excitability (Nitsche and Paulus, 2001) as well as for ERPs in the somatosensory (Matsunaga et al., 2004; Dieckhofer et al., 2006; Antal et al., 2008), and auditory modalities (Zaehle et al., 2011a). In agreement with these findings, our results revealed polarity-specific effects of anodal and cathodal tDCS on cortical activity.

Moreover, during the retention of the visuo-spatial information, we found a significant decrease in oscillatory power in the alpha band for the cathodal tDCS over the parietal cortex. Generally, WM operations have been related to oscillatory brain activity in multiple frequency bands, including the theta $(4-8 \mathrm{~Hz})$, alpha $(8-12 \mathrm{~Hz})$, and beta $(12-30 \mathrm{~Hz})$ range (Klimesch et al., 2005). In particular the performance in visual WM tasks has been specifically associated with alterations in event-related alpha band activity (Pesonen et al., 2007). In this regard alpha activity is assumed to reflect a general inhibition of non-task relevant areas (Klimesch, 1999) and may index the degree of inhibition necessary during internally, as opposed to externally, directed attention (Cooper et al., 2003). Furthermore, alpha activity increases during the retention interval of memory tasks, when participants need to keep in mind several items after encoding, and later responded to a probe (Klimesch, 1999; Busch and Herrmann, 2003; Sauseng et al., 2005; Klimesch et al., 2007). Moreover, alpha power increases with increasing number of items to be remembered (Klimesch, 1999; Jensen et al., 2002; Schack and Klimesch, 2002). Thus our finding of an increase in alpha activity by means of cathodal tDCS might be directly related to the increased performance of the participants in the WM task.

Previously, we showed that tDCS over the left DLPFC induces altered WM performance by modulating its alpha activity. In particular, we demonstrated that cathodal tDCS of the left DLPFC decreases alpha activity over posterior scalp locations (Zaehle et al., 2011b). This effect is in accordance with the present data showing decreased posterior alpha activity after cathodal tDCS of the right parietal lobe. Even though we previously interpreted the modulatory effects of tDCS on WM to be specifically related to the responsiveness of the left DLPFC, it can be assumed that altered local cortical excitability in one part of the responsible network influences the whole neural network associated with WM functions beyond the site of stimulation leading to comparable electrophysiological effects. Thus, the reduction of alpha band activity after cathodal tDCS of either the left DLPFC or the right posterior parietal cortex might be related to general

\section{REFERENCES}

Accornero, N., Li Voti, P., La Riccia, M., and Gregori, B. (2007). Visual evoked potentials modulation during direct current cortical polarization. Exp. Brain Res. 178, 261-266.

Alescio-Lautier, B., Michel, B. F., Herrera, C., Elahmadi, A., Chambon, C., Touzet, C., and Paban, V. (2007). Visual and visuospatial short-term memory in mild cognitive impairment and Alzheimer disease: role of attention. Neuropsychologia 45, 1948-1960.

Antal, A., Brepohl, N., Poreisz, C., Boros, K., Csifcsak, G., and Paulus, W. (2008). Transcranial direct current stimulation over somatosensory cortex decreases experimentally induced acute pain perception. Clin. J. Pain 24, 56-63.

Antal, A., Kincses, T. Z., Nitsche, M. A., Bartfai, O., and Paulus, W. (2004). Excitability changes induced in the

effects of the tDCS on the underlying fronto-parietal network involved in visuo-spatial WM. Indeed, widespread tDCS-induced changes in cortical activity have been demonstrated by a previous neuroimaging study (Keeser et al., 2011). Moreover, it can be demonstrated by simulation approaches, that the mounting of the current electrode at parietal electrode sites P8/P10 induces currents not only to posterior (parietal and occipital) brain areas, but also to the temporal and frontal cortices. Thus, it is likely that by influencing one component of the WM network, the electrical stimulation had an influence on the functioning of the entire WM system.

In the present study we also revealed tDCS-related effects for stimuli attended in the right hemifield, i.e., ipsilateral to the active tDCS stimulation. Even though EEG studies consistently report parietal contralateral activity during visual WM tasks to be strongly lateralized (Klaver et al., 1999; Vogel and Machizawa, 2004), functional magnetic resonance imaging (fMRI) also emphasizes bilateral parietal BOLD activation (Robitaille et al., 2010). It has been proposed that this discrepancy might relate to the different temporal resolutions of both methods. It might be that the mnemonic representations of the parietal cortex are initially lateralized, but become more bilateral over time within one trial. Thus, give the good temporal resolution of EEG data, the lateralized ERP components are more suitable to detect this specific differences and $\mathrm{AMRI}$ is not able to resolve this transient effects (Robitaille et al., 2010). However, based on these divergent reports we cannot rule out bilateral involvement of the parietal cortex during the particular paradigm used in the present study. Consequently, the involvements of the ipsilateral hemisphere might explain the observed electrophysiological and behavioral effects on stimuli that have been attended in the right hemifield.

In summary the present study shows that tDCS of the parietal cortex can change the organized cortical activity associated with visuo-spatial WM in concert with systematic alterations of WM performance. To our knowledge, this is the first study investigating the effects of parietal tDCS on electrophysiological brain activity in the context of a visuo-spatial WM task. The results of the study will provide a better understanding of the neuromodulatory effects of tDCS and demonstrate its potential at fostering knowledge for therapeutic application of tDCS in neuropsychiatric diseases.

\section{ACKNOWLEDGMENTS}

This study was supported by the Deutsche Forschungsgemeinschaft (SFB/TR31-TPA9; K. Heimrath, T. Zaehle) and (DFG Mu1364/4-1; A. Becke).

human primary visual cortex by transcranial direct current stimulation: direct electrophysiological evidence. Invest. Ophthalmol. Vis. Sci. 45, 702-707.

Antal, A., Kincses, T. Z., Nitsche, M. A., and Paulus, W. (2003). Manipulation of phosphene thresholds by transcranial direct current stimulation in man. Exp. Brain Res. 150, 375-378.

Baddeley, A. (1992). Working memory. Science 255, 556-559.
Barch, D. M., Sheline, Y. I., Csernansky, J. G., and Snyder, A. Z (2003). Working memory and prefrontal cortex dysfunction: specificity to schizophrenia compared with major depression. Biol. Psychiatry 53, 376-384.

Berryhill, M. E., Wencil, E. B., Branch Coslett, H., and Olson, I. R. (2010). A selective working memory impairment after transcranial direct current stimulation to the right parietal lobe. Neurosci. Lett. 479, 312-316. 
Bindman, L. J., Lippold, O. C., and Redfearn, J. W. (1962). Long-lasting changes in the level of the electrical activity of the cerebral cortex produced bypolarizing currents. Nature 196, 584-585.

Birks, J. (2006). Cholinesterase inhibitors for Alzheimer's disease. Cochrane Database Syst. Rev. CD005593.

Boggio, P. S., Ferrucci, R., Mameli, F., Martins, D., Martins, O., Vergari, M., Tadini, L., Scarpini, E., Fregni, F., and Priori, A. (2011). Prolonged visual memory enhancement after direct current stimulation in Alzheimer's disease. Brain Stimul. [Epub ahead of print].

Boggio, P. S., Ferrucci, R., Rigonatti, S. P., Covre, P., Nitsche, M., PascualLeone, A., and Fregni, F. (2006). Effects of transcranial direct current stimulation on working memory in patients with Parkinson's disease. J. Neurol. Sci. 249, 31-38.

Boggio, P. S., Khoury, L. P., Martins, D. C., Martins, O. E., De Macedo, E. C., and Fregni, F. (2009). Temporal cortex direct current stimulation enhances performance on a visual recognition memory task in Alzheimer disease. J. Neurol. Neurosurg. Psychiatr. 80, 444-447.

Boggio, P. S., Nunes, A., Rigonatti, S. P., Nitsche, M. A., Pascual-Leone, A., and Fregni, F. (2007). Repeated sessions of noninvasive brain DC stimulation is associated with motor function improvement in stroke patients. Restor. Neurol. Neurosci. 25, 123-129.

Boggio, P. S., Rigonatti, S. P., Ribeiro, R. B., Myczkowski, M. L., Nitsche, M. A., Pascual-Leone, A., and Fregni, F. (2008). A randomized, doubleblind clinical trial on the efficacy of cortical direct current stimulation for the treatment of major depression. Int. J. Neuropsychopharmacol. 11, 249-254.

Boggio, P. S., Zaghi, S., Villani, A. B., Fecteau, S., Pascual-Leone, A., and Fregni, F. (2010). Modulation of risk-taking in marijuana users by transcranial direct current stimulation (tDCS) of the dorsolateral prefrontal cortex (DLPFC). Drug Alcohol Depend. 112, 220-225.

Bolognini, N., Pascual-Leone, A., and Fregni, F. (2009). Using non-invasive brain stimulation to augment motor training-induced plasticity. J. Neuroeng. Rehabil. 6, 8.

Brunoni, A. R., Nitsche, M. A., Bolognini, N., Bikson, M., Wagner, T., Merabet, L., Edwards, D. J., Valero-Cabre, A., Rotenberg, A.,
Pascual-Leone, A., Ferrucci, R., Priori, A., Boggio, P. S., and Fregni, F. (2011). Clinical research with transcranial direct current stimulation (tDCS): challenges and future directions. Brain Stimul. [Epub ahead of print].

Busch, N. A., and Herrmann, C. S. (2003). Object-load and featureload modulate EEG in a shortterm memory task. Neuroreport 14, 1721-1724.

Callicott, J. H., Mattay, V. S., Bertolino, A., Finn, K., Coppola, R., Frank, J. A., Goldberg, T. E., and Weinberger, D. R. (1999). Physiological characteristics of capacity constraints in working memory as revealed by functional MRI. Cereb. Cortex 9, 20-26.

Cooper, N. R., Croft, R. J., Dominey, S. J., Burgess, A. P., and Gruzelier, J. H. (2003). Paradox lost? Exploring the role of alpha oscillations during externally vs. internally directed attention and the implications for idling and inhibition hypotheses. Int. J. Psychophysiol. 47, 65-74.

Corbetta, M., Patel, G., and Shulman, G. L. (2008). The reorienting system of the human brain: from environment to theory of mind. Neuron 58 , 306-324.

Courtney, S. M., Petit, L., Haxby, J. V., and Ungerleider, L. G. (1998). The role of prefrontal cortex in working memory: examining the contents of consciousness. Philos. Trans. R. Soc. Lond. B Biol. Sci. 353, 1819-1828.

Cowan, N. (2001). The magical number 4 in short-term memory: a reconsideration of mental storage capacity. Behav. Brain Sci. 24, 87-114; discussion 114-185.

Dieckhofer, A., Waberski, T. D., Nitsche, M., Paulus, W., Buchner, H., and Gobbele, R. (2006). Transcranial direct current stimulation applied over the somatosensory cortex differential effect on low and high frequency SEPs. Clin. Neurophysiol. 117, 2221-2227.

Elmer, S., Burkard, M., Renz, B., Meyer, M., and Jancke, L. (2009). Direct current induced short-term modulation of the left dorsolateral prefrontal cortex while learning auditory presented nouns. Behav. Brain Funct. 5, 29.

Ferrucci, R., Mameli, F., Guidi, I., Mrakic-Sposta, S., Vergari, M., Marceglia, S., Cogiamanian, F., Barbieri, S., Scarpini, E., and Priori, A. (2008a). Transcranial direct current stimulation improves recognition memory in Alzheimer disease. Neurology 71, 493-498.

Ferrucci, R., Marceglia, S., Vergari, M., Cogiamanian, F., Mrakic-Sposta,
S., Mameli, F., Zago, S., Barbieri, S., and Priori, A. (2008b). Cerebellar transcranial direct current stimulation impairs the practicedependent proficiency increase in working memory. J. Cogn. Neurosci. 20, 1687-1697.

Fox, D. (2011). Neuroscience: brain buzz. Nature 472, 156-158.

Fregni, F., Boggio, P. S., Nitsche, M. Bermpohl, F., Antal, A., Feredoes, E., Marcolin, M. A., Rigonatti, S. P., Silva, M. T., Paulus, W., and PascualLeone, A. (2005). Anodal transcranial direct current stimulation of prefrontal cortex enhances working memory. Exp. Brain Res. 166, 23-30.

Fregni, F., Boggio, P. S., Santos, M. C. Lima, M., Vieira, A. L., Rigonatti, S. P., Silva, M. T., Barbosa, E. R., Nitsche, M. A., and Pascual-Leone, A. (2006). Noninvasive cortical stimulation with transcranial direct current stimulation in Parkinson's disease. Mov. Disord. 21, 1693-1702.

Funahashi, S., Bruce, C. J., and Goldman-Rakic, P. S. (1993). Dorsolateral prefrontal lesions and oculomotor delayed-response performance: evidence for mnemonic "scotomas." J. Neurosci. 13 1479-1497.

Gartside, I. B. (1968). Mechanisms of sustained increases of firing rate of neurons in the rat cerebral cortex after polarization: reverberating circuits or modification of synaptic conductance? Nature 220, 382-383.

Hammer, A., Vielhaber, S., RodriguezFornells, A., Mohammadi, B., and Munte, T. F. (2011). A neurophysiological analysis of working memory in amyotrophic lateral sclerosis. Brain Res. 1421, 90-99.

Hattori, Y., Moriwaki, A., and Hori, Y. (1990). Biphasic effects of polarizing current on adenosine-sensitive generation of cyclic AMP in rat cerebral cortex. Neurosci. Lett. 116, 320-324.

Hautzel, H., Mottaghy, F. M., Schmidt, D., Zemb, M., Shah, N. J., MullerGartner, H. W., and Krause, B. J. (2002). Topographic segregation and convergence of verbal, object, shape and spatial working memory in humans. Neurosci. Lett. 323, 156-160.

Huntley, J. D., and Howard, R. J. (2010). Working memory in early Alzheimer's disease: a neuropsychological review. Int. J. Geriatr. Psychiatry 25, 121-132.

Jacobson, L., Koslowsky, M., and Lavidor, M. (2012). tDCS polarity effects in motor and cognitive domains: a meta-analytical review. Exp. Brain Res. 216, 1-10.
Jasper, H. H. (1958). The ten-twenty electrode system of the International Federation. Electroencephalogr. Clin. Neurophysiol. 10, 371-375.

Jensen, O., Gelfand, J., Kounios, J., and Lisman, J. E. (2002). Oscillations in the alpha band $(9-12 \mathrm{~Hz})$ increase with memory load during retention in a short-term memory task. Cereb. Cortex 12, 877-882.

Jo, J. M., Kim, Y. H., Ko, M. H., Ohn, S. H., Joen, B., and Lee, K. H. (2009). Enhancing the working memory of stroke patients using tDCS. Am. J. Phys. Med. Rehabil. 88, 404-409.

Keeser, D., Meindl, T., Bor, J., Palm, U., Pogarell, O., Mulert, C., Brunelin, J., Moller, H. J., Reiser, M., and Padberg, F. (2011). Prefrontal transcranial direct current stimulation changes connectivity of resting-state networks during fMRI. J. Neurosci. 31, 15284-15293.

Klaver, P., Talsma, D., Wijers, A. A. Heinze, H. J., and Mulder, G. (1999). An event-related brain potential correlate of visual short-term memory. Neuroreport 10, 2001-2005.

Klimesch, W. (1999). EEG alpha and theta oscillations reflect cognitive and memory performance: a review and analysis. Brain Res. Brain Res. Rev. 29, 169-195.

Klimesch, W., Sauseng, P., and Hanslmayr, S. (2007). EEG alpha oscillations: the inhibition-timing hypothesis. Brain Res. Rev. 53, 63-88.

Klimesch, W., Schack, B., and Sauseng, P. (2005). The functional significance of theta and upper alpha oscillations. Exp. Psychol. 52, 99-108.

Lee, E. Y., Cowan, N., Vogel, E. K., Rolan, T., Valle-Inclan, F., and Hackley, S. A. (2010). Visual working memory deficits in patients with Parkinson's disease are due to both reduced storage capacity and impaired ability to filter out irrelevant information. Brain 133, 2677-2689.

Lees, A. J., and Smith, E. (1983). Cognitive deficits in the early stages of Parkinson's disease. Brain 106(Pt 2), 257-270.

Liebetanz, D., Nitsche, M. A., Tergau, F., and Paulus, W. (2002). Pharmacological approach to the mechanisms of transcranial DCstimulation-induced after-effects of human motor cortex excitability. Brain 125, 2238-2247.

Loui, P., Hohmann, A., and Schlaug, G. (2010). Inducing disorders in pitch perception and production: a reverse-engineering approach. Proc. Meet. Acoust. 9, 50002.

Marder, S. R. (2006). Drug initiatives to improve cognitive function. J. 
Clin. Psychiatry 67(Suppl. 9), 31-35; discussion 36-42.

Maris, E., Schoffelen, J. M., and Fries, P. (2007). Nonparametric statistical testing of coherence differences. $J$. Neurosci. Methods 163, 161-175.

Marshall, L., Molle, M., Siebner, H. R., and Born, J. (2005). Bifrontal transcranial direct current stimulation slows reaction time in a working memory task. BMC Neurosci. 6, 23. doi:10.1186/1471-2202-6-23

Matsunaga, K., Nitsche, M. A., Tsuji, S., and Rothwell, J. C. (2004). Effect of transcranial DC sensorimotor cortex stimulation on somatosensory evoked potentials in humans. Clin. Neurophysiol. 115, 456-460.

McAllister, T. W., Flashman, L. A., McDonald, B. C., Ferrell, R. B., Tosteson, T. D., Yanofsky, N. N., Grove, M. R., and Saykin, A. J. (2011). Dopaminergic challenge with bromocriptine one month after mild traumatic brain injury: altered working memory and BOLD response. J. Neuropsychiatry Clin. Neurosci. 23, 277-286.

McGurk, S. R., Twamley, E. W., Sitzer, D. I., McHugo, G. J., and Mueser, K. T. (2007). A meta-analysis of cognitive remediation in schizophrenia. Am. J. Psychiatry 164, 1791-1802.

McNab, F., and Klingberg, T. (2008). Prefrontal cortex and basal ganglia control access to working memory. Nat. Neurosci. 11, 103-107.

Miranda, P. C., Lomarev, M., and Hallett, M. (2006). Modeling the current distribution during transcranial direct current stimulation. Clin. Neurophysiol. 117, 1623-1629.

Monti, A., Cogiamanian, F., Marceglia, S., Ferrucci, R., Mameli, F., MrakicSposta, S., Vergari, M., Zago, S., and Priori, A. (2008). Improved naming after transcranial direct current stimulation in aphasia. J. Neurol. Neurosurg. Psychiatr. 79, 451-453.

Neuling, T., Wagner, S., Wolters, C. H., Zaehle, T., and Herrmann, C. S. (2012). Finite element model predicts current density distribution for clinical applications of tDCS and tACS. Front. Psychiatry.

Nitsche, M. A., Boggio, P. S., Fregni, F., and Pascual-Leone, A. (2009). Treatment of depression with transcranial direct current stimulation (tDCS): a review. Exp. Neurol. 219, 14-19.

Nitsche, M. A., Cohen, L. G., Wassermann, E. M., Priori, A., Lang, N., Antal, A., Paulus, W., Hummel, F.,
Boggio, P. S., Fregni, F., and PascualLeone, A. (2008). Transcranial direct current stimulation: State of the art 2008. Brain Stimul. 1, 206-223.

Nitsche, M. A., Liebetanz, D., Lang, N., Antal, A., Tergau, F., and Paulus, W. (2003). Safety criteria for transcranial direct current stimulation (tDCS) in humans. Clin Neurophysiol 114, 2220-2222.

Nitsche, M. A., and Paulus, W. (2000). Excitability changes induced in the human motor cortex by weak transcranial direct current stimulation. $J$. Physiol. (Lond.) 527(Pt 3), 633-639.

Nitsche, M. A., and Paulus, W. (2001). Sustained excitability elevations induced by transcranial DC motor cortex stimulation in humans. Neurology 57, 1899-1901.

Nitsche, M. A., and Paulus, W. (2011). Transcranial direct current stimulation - update 2011. Restor. Neurol. Neurosci. 29, 463-492.

Nystrom, L. E., Braver, T. S., Sabb, F. W., Delgado, M. R., Noll, D. C., and Cohen, J. D. (2000). Working memory for letters, shapes, and locations: fMRI evidence against stimulus-based regional organization in human prefrontal cortex. Neuroimage 11, 424-446.

Ohn, S. H., Park, C. I., Yoo, W. K., Ko, M. H., Choi, K. P., Kim, G. M., Lee, Y. T., and Kim, Y. H. (2008). Time-dependent effect of transcranial direct current stimulation on the enhancement of working memory. Neuroreport 19, 43-47.

Olson, I. R., and Berryhill, M. (2009). Some surprising findings on the involvement of the parietal lobe in human memory. Neurobiol. Learn. Mem. 91, 155-165.

Owen, A. M., Iddon, J. L., Hodges, J. R., Summers, B. A., and Robbins, T. W. (1997). Spatial and nonspatial working memory at different stages of Parkinson's disease. Neuropsychologia 35, 519-532.

Owen, A. M., McMillan, K. M., Laird, A. R., and Bullmore, E. (2005). Nback working memory paradigm: a meta-analysis of normative functional neuroimaging studies. Hum. Brain Mapp. 25, 46-59.

Pesonen, M., Hamalainen, H., and Krause, C. M. (2007). Brain oscillatory $4-30 \mathrm{~Hz}$ responses during a visual $\mathrm{n}$-back memory task with varying memory load. Brain Res. 1138, 171-177.

Priori, A. (2003). Brain polarization in humans: a reappraisal of an old tool for prolonged non-invasive modulation of brain excitability. Clin. Neurophysiol. 114, 589-595.

Priori, A., Berardelli, A., Rona, S. Accornero, N., and Manfredi, M. (1998). Polarization of the human motor cortex through the scalp. Neuroreport 9, 2257-2260.

Purpura, D. P., Scarff, T., and McMurtry, J. G. (1965). Intracellular study of internuclear inhibition in ventrolateral thalamic neurons. J. Neurophysiol. 28, 487-496.

Robitaille, N., Marois, R., Todd, J., Grimault, S., Cheyne, D., and Jolicoeur, P. (2010). Distinguishing between lateralized and nonlateralized brain activity associated with visual short-term memory: fMRI MEG, and EEG evidence from the same observers. Neuroimage 53 1334-1345.

Rose, E. J., and Ebmeier, K. P. (2006). Pattern of impaired working memory during major depression. J. Affect. Disord. 90, 149-161.

Sauseng, P., Klimesch, W., Doppelmayr, M., Pecherstorfer, T., Freunberger, R., and Hanslmayr, S. (2005). EEG alpha synchronization and functional coupling during topdown processing in a working memory task. Hum. Brain Mapp. 26, 148-155.

Schack, B., and Klimesch, W. (2002). Frequency characteristics of evoked and oscillatory electroencephalic activity in a human memory scanning task. Neurosci. Lett. 331, 107-110.

Smith, E. E., and Jonides, J. (1997). Working memory: a view from neuroimaging. Cogn. Psychol. 33, 5-42.

Todd, J. J., and Marois, R. (2004). Capacity limit of visual short-term memory in human posterior parietal cortex. Nature 428, 751-754.

Vines, B. W., Schnider, N. M., and Schlaug, G. (2006). Testing for causality with transcranial direct current stimulation: pitch memory and the left supramarginal gyrus. Neuroreport 17, 1047-1050.

Vogel, E. K., and Machizawa, M. G. (2004). Neural activity predicts individual differences in visual working memory capacity. Nature 428 748-751.

Wager, T. D., and Smith, E. E. (2003). Neuroimaging studies of working memory: a meta-analysis. Cogn. Affect. Behav. Neurosci. 3, 255-274.
Wallace, D. L., Vytlacil, J. J., Nomura, E. M., Gibbs, S. E., and D'esposito, M. (2011). The dopamine agonist bromocriptine differentially affects fronto-striatal functional connectivity during working memory. Front. Hum. Neurosci. 5:32. doi:10.3389/fnhum.2011.00032

You, D. S., Kim, D. Y., Chun, M. H., Jung, S. E., and Park, S. J. (2011). Cathodal transcranial direct current stimulation of the right Wernicke's area improves comprehension in subacute stroke patients. Brain Lang. 119 , 1-5.

Zaehle, T., Beretta, M., Jancke, L., Herrmann, C. S., and Sandmann, P. (2011a). Excitability changes induced in the human auditory cortex by transcranial direct current stimulation: direct electrophysiological evidence. Exp. Brain Res. 215, 135-140.

Zaehle, T., Sandmann, P., Thorne, J. D., Jancke, L., and Herrmann, C. S. (2011b). Transcranial direct current stimulation of the prefrontal cortex modulates working memory performance: combined behavioural and electrophysiological evidence. BMC Neurosci. 12, 2. doi:10.1186/1471-2202-12-2

Conflict of Interest Statement: The authors declare that the research was conducted in the absence of any commercial or financial relationships that could be construed as a potential conflict of interest.

Received: 15 March 2012; paper pending published: 13 April 2012; accepted: 23 May 2012; published online: 20 June 2012.

Citation: Heimrath $K$, Sandmann $P$, Becke A, Müller NG and Zaehle T (2012) Behavioral and electrophysiological effects of transcranial direct current stimulation of the parietal cortex in a visuo-spatial working memory task. Front. Psychiatry 3:56. doi: 10.3389/fpsyt.2012.00056

This article was submitted to Frontiers in Neuropsychiatric Imaging and Stimulation, a specialty of Frontiers in Psychiatry. Copyright $\odot 2012$ Heimrath, Sandmann, Becke, Müller and Zaehle. This is an open-access article distributed under the terms of the Creative Commons Attribution Non Commercial License, which permits non-commercial use, distribution, and reproduction in other forums, provided the original authors and source are credited. 